\title{
Laboratory simulations of solar prominence eruptions*
}

\author{
P. M. Bellan ${ }^{\dagger}$ and J. F. Hansen \\ California Institute of Technology, Pasadena, California 91125
}

(Received 12 November 1997; accepted 13 January 1998)

\begin{abstract}
Spheromak technology is exploited to create laboratory simulations of solar prominence eruptions. It is found that the initial simulated prominences are arched, but then bifurcate into twisted secondary structures which appear to follow fringing field lines. A simple model explains many of these topological features in terms of the trajectories of field lines associated with relaxed states, i.e., states satisfying $\nabla \times \mathbf{B}=\lambda \mathbf{B}$. This model indicates that the field line concept is more fundamental than the flux tube concept because a field line can always be defined by specifying a starting point whereas attempting to define a flux tube by specifying a starting cross section typically works only if $\lambda$ is small. The model also shows that, at least for plasma evolving through a sequence of force-free states, the oft-used line-tying concept is in error. Contrary to the predictions of line-tying, direct integration of field line trajectories shows explicitly that when $\lambda$ is varied, both ends of field lines intersecting a flux-conserving plane do not remain anchored to fixed points in that plane. Finally, a simple explanation is provided for the $\mathrm{S}$-shaped magnetic structures often seen on the sun; the $\mathrm{S}$ shape is shown to be an automatic consequence of field line arching and the parallelism between magnetic field and current density for force-free states. (C) 1998 American Institute of Physics. [S1070-664X(98)92705-7]
\end{abstract}

\section{INTRODUCTION}

Prominences are large arch-shaped plasma configurations emanating from the solar surface. ${ }^{1}$ Prominences typically remain stable for many days but can then erupt in a few minutes, ejecting plasma and magnetic fields; the cause of this dramatic behavior is not well understood. This paper presents preliminary results from an experiment designed to simulate prominence dynamics in the laboratory and also presents a simple model interpreting the main features of these results.

\section{BRIEF OVERVIEW OF PROMINENCES}

Typical solar prominences are shown schematically in Fig. 1; the different appearance of prominences protruding from the edge (limb) of the sun compared to prominences on the solar disk is known to be a consequence of the observer's line of sight. Prominences extending from the limb and thus viewed from the side have an arched shape and appear to be composed of twisted magnetic field lines or twisted ribbons of magnetic flux. In contrast, prominences on the solar surface and thus viewed from above typically have a forward or reverse S shape (sigmoid). Rust and $\mathrm{Kumar}^{2}$ proposed that reverse $\mathrm{S}$ corresponds to negative magnetic helicity while forward $S$ corresponds to positive magnetic helicity.

Vrsnak et al. ${ }^{3}$ analyzed photographs of a large number of limb prominences before, during, and after eruption, and found a correlation between twist and instability. Vrsnak et al.'s data shows that prominences tend to erupt when the nominal pitch angle of the twisted magnetic field exceeds a threshold of approximately $45^{\circ}$.

\footnotetext{
*Paper eMopI2-2 Bull. Am. Phys. Soc. 42, 1871 (1997).

${ }^{\dagger}$ Invited speaker.
}

Order of magnitude analysis ${ }^{4}$ shows that prominences are low $\beta$ and quasi-steady so that they are in a force-free equilibrium, i.e., satisfy $\mathbf{J} \times \mathbf{B}=0$. Thus the current and magnetic field must be parallel and from Ampere's law the magnetic field must therefore satisfy

$$
\nabla \times \mathbf{B}=\lambda \mathbf{B},
$$

where $\lambda(\mathbf{r})$ is some scalar function. The divergence of Eq. (1) shows that $\lambda$ is constant along a field line. If $\lambda=0$, the magnetic field is a vacuum field $\mathbf{B}=\nabla \chi$ (also called potential field) and has no twist, but if $\lambda \neq 0$ then Eq. (1) shows that the magnetic field will be twisted. Twisted prominences have currents, contain magnetic helicity, and have free energy compared to untwisted (vacuum field) prominences satisfying the same boundary conditions. Nakagawa et al. ${ }^{4}$ assumed that $\lambda$ is uniform over the extent of a prominence in which case analytic solutions of Eq. (1) can be found. The assumption of uniform $\lambda$ is precisely the criterion for the Taylor state. ${ }^{5,6}$ Heyvaerts and Priest ${ }^{7}$ modeled prominence arcades using a quasi-harmonic Taylor state while Browning ${ }^{8}$ and Rust and Kumar ${ }^{9}$ modeled individual prominences as straight force-free cylinders in Taylor state equilibria with fields given by the axisymmetric, axially invariant solution to Eq. (1),

$$
B_{z}=\bar{B} J_{0}(\lambda r), \quad B_{\theta}=\bar{B} J_{1}(\lambda r) .
$$

Equation (2) is known ${ }^{6}$ to describe both spheromak and reversed field pinch equilibria reasonably well.

The plasma and magnetic field ejected by an erupting prominence propagate into interplanetary space and sometimes intercept the earth's magnetosphere, generating magnetic storms. These propagating interplanetary magnetic disturbances have been clearly identified by spacecraft ${ }^{10}$ and are 


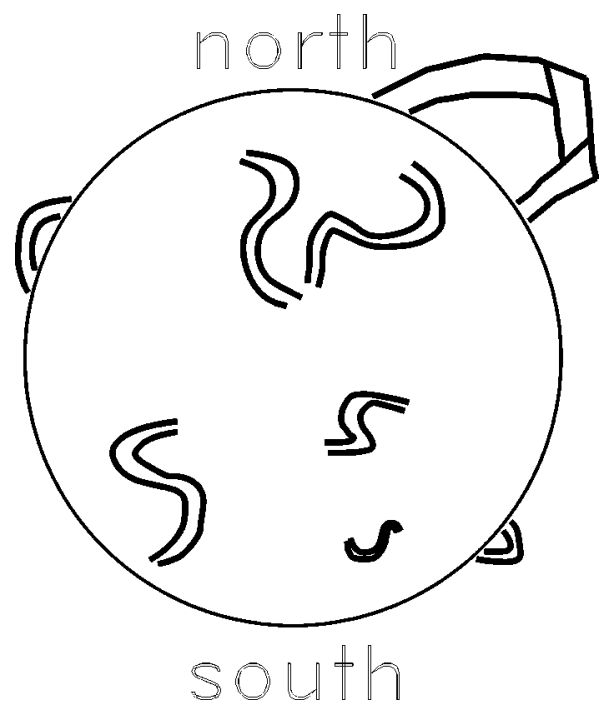

FIG. 1. Sketch of typical prominences showing backwards $S$ shapes in the north solar hemisphere, forwards $\mathrm{S}$ in south hemisphere, and arched, twisted prominences on limb.

called magnetic clouds. The spacecraft measurements ${ }^{10}$ show that the magnetic cloud fields are in excellent agreement with Eq. (2); i.e., magnetic clouds are Taylor states. Magnetic cloud observations have been correlated ${ }^{11}$ with the eruption of solar prominences and, in particular, it has been found that a magnetic cloud observation near earth is typically preceded by a prominence eruption about $80-100 \mathrm{~h}$ earlier (the time of flight from Sun to Earth). There is also excellent correlation $^{11}$ between magnetic cloud handedness and the handedness of the erupting prominence: prominences with left-handed twist spawn magnetic clouds with left-handed twist while right-handed prominences spawn right-handed clouds. This identification has been simplified by the as yet unexplained observation ${ }^{12}$ that prominences and related structures in the north solar hemisphere tend to be left handed while prominences in the south solar hemisphere tend to be right handed. Thus a left-handed magnetic cloud observed near earth is typically preceded by a prominence eruption in the north solar hemisphere and vice versa for right-handed clouds. Rust and Kumar ${ }^{13}$ also noted that erupting prominences in the north solar hemisphere typically have a reverse $\mathrm{S}$ shape while those in the south hemisphere typically have forward $\mathrm{S}$.

\section{SIMILARITY OF PROMINENCE ERUPTIONS TO SPHEROMAK FORMATION}

Woltjer $^{14}$ showed that both magnetic energy and magnetic helicity are exactly conserved in a nondissipative low $\beta$ plasma and conjectured that in a slightly dissipative plasma, magnetic helicity decays much more slowly than magnetic energy. Minimization of magnetic energy is therefore constrained by the requirement of helicity conservation. By posing this constrained energy minimization as a variational problem, Woltjer found the resulting magnetic field to be prescribed by Eq. (1) with $\lambda$ spatially uniform. Taylor ${ }^{5}$ extended Woltjer's analysis by arguing that dissipative magnetic turbulence (including reconnection) conserves mag-

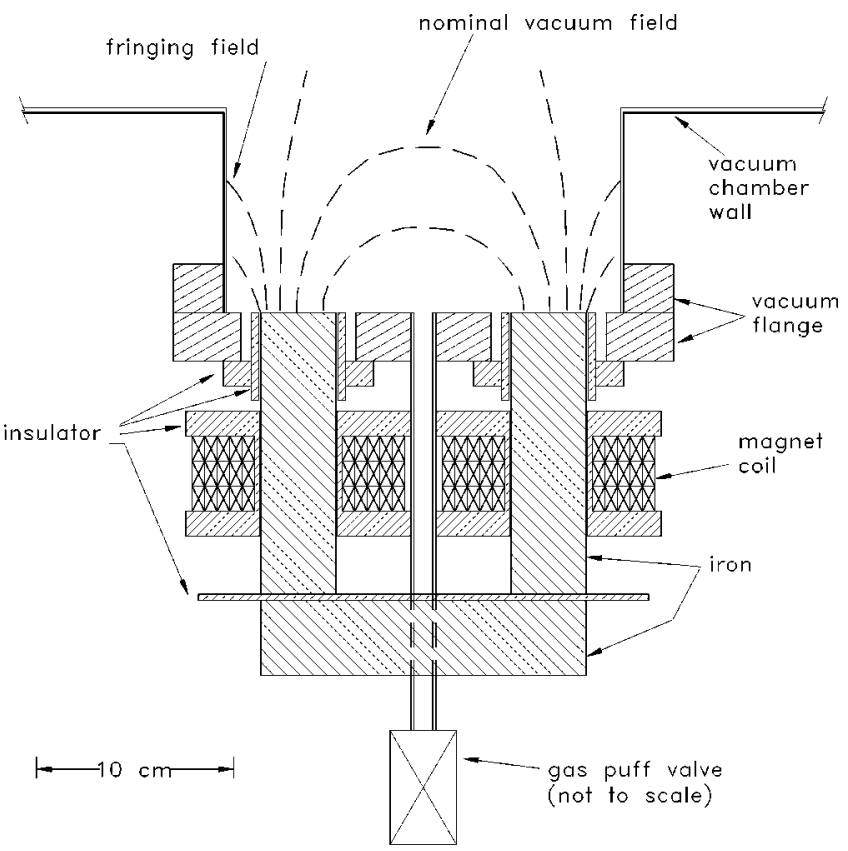

FIG. 2. Experimental configuration. Note fringing fields; neutral lines (separatrices) between oppositely directed fields occur between the two magnet poles and also where fringing fields reverse direction.

netic helicity almost perfectly so that turbulence would invariably cause a plasma to relax to uniform $\lambda$ equilibria described by Eq. (1). These equilibria are called Taylor or relaxed states.

Spheromaks ${ }^{15}$ are toroidal Taylor states contained inside a close-fitting, simply connected conducting shell and have all magnetic fields provided either by internal currents or image currents in the shell wall. Spheromaks have a formation threshold determined by magnetic pitch angle, form from attached flux tubes via a magnetic reconnection process that conserves helicity while minimizing magnetic energy, and involve the evolution of quasi-static force-free equilibria both before and after formation.

Our laboratory simulation is based on the premise that spheromak formation and prominence eruption are analogous. The justification is that both processes involve the twisting up of low $\beta$ attached flux tubes to the point of instability. The equilibria before and after instability are relaxed states satisfying Eq. (1). When the current twists the configuration beyond a critical threshold, it becomes unstable and ejects a detached spheromak or magnetic cloud which carries away the excess helicity.

\section{DESIGN AND PRELIMINARY RESULTS OF THE LABORATORY PROMINENCE SIMULATION}

The simulation experiment uses spheromak technology, but has both geometry and boundary conditions specifically arranged to model prominences. Prominence geometry is provided by a horseshoe electromagnet which forms initial arched vacuum field lines as shown in Fig. 2. The electromagnet is powered by an electrolytic capacitor bank, has an $8 \mathrm{~ms}$ rise time, and is gapped so that its pole faces can be biased to different electric potentials. The magnetic field at 
the $3.7 \mathrm{~cm}$ diameter magnet pole faces is adjusted to $\approx 0.3 \mathrm{~T}$ corresponding to a magnetic flux $\Phi \approx 0.3 \mathrm{mWb}$. When $\Phi$ has reached this value, a fast gas valve ( $25 \mu$ s opening time) puffs approximately 25 Torr-liters of hydrogen into the region between the two magnet poles. Then a $60 \mu \mathrm{F}$ capacitor charged to $6 \mathrm{kV}$ is connected by a fast ignitron switch across the magnet poles. The high voltage causes the hydrogen to break down, forming plasma which acts as a near shortcircuit across the magnet poles. The capacitor bank discharges through this circuit and a current $I$ flows along the vacuum magnetic field lines. The self-magnetic field of the current $I$ is expected to twist up the field lines giving a twisted arch-shaped flux tube.

An essential requirement for simulation is to have a ground-plane boundary corresponding to the solar surface; the actual solar prominence has its footpoints in this plane and projects into infinite half-space. A reasonable approximation to semi-infinite geometry is achieved by using a vacuum chamber much larger (1.4 m diameter, $2 \mathrm{~m}$ long) than the prominence simulator $(0.12 \mathrm{~m}$ between magnet poles) so that the initial simulated prominence is far from all walls except the wall which acts as the ground plane. The effect of the far walls is negligible until the simulated prominence has expanded to fill the vacuum chamber.

The experimental time scale must be short compared to resistive times so that the plasma acts as a flux conserver. A suitably short time scale is achieved by minimizing the series inductance in the gun-capacitor bank circuit. The total series inductance is $<0.5 \mu \mathrm{H}$ giving a current rise time of $\simeq 5 \mu \mathrm{s}$. The experiment duration is of the order of $10 \mu \mathrm{s}$. The plasma electron temperature is assumed to be $5-10 \mathrm{eV}$ giving a Spitzer resistivity $\eta \approx 3 \times 10^{-5}-9 \times 10^{-5} \Omega \mathrm{m}$. The classical resistive diffusion time is of the order of $\tau_{R} \sim \sqrt{\mu_{0} r / 4 \eta}$ $\sim 10 \mathrm{~ms}$ using $r=3 \mathrm{~cm}$ so the experimental time is much shorter than the resistive diffusion time. Another important characteristic time is the Alfven time, the time of flight for an Alfven wave to traverse the dimensions of the simulated flux tube. Although the density has not yet been measured in this experiment, previous spheromak experiments ${ }^{16}$ with similar operating conditions had densities $n=10^{19}-10^{21}$ $\mathrm{m}^{-3}$. Assuming a nominal density $n=10^{20} \mathrm{~m}^{-3}$ and using the $0.3 \mathrm{~T}$ magnetic field at the magnet pole as the nominal magnetic field gives the characteristic Alfven velocity for a hydrogen plasma to be $v_{A} \sim 6 \times 10^{5} \mathrm{~m} \mathrm{~s}^{-1}$. The time for an Alfven wave to traverse the $0.3 \mathrm{~m}$ nominal length of a simulated prominence will be $\tau_{A} \sim 0.5 \mu \mathrm{s}$ and the time to cross the $2 \mathrm{~cm}$ nominal minor radius will be $\tau_{A} \sim 30 \mathrm{~ns}$. Thus the experimental duration is longer than the Alfven time and so the plasma should be in a sequence of force-free equilibrium states. The Lundquist number $S=\tau_{R} / \tau_{A}$ is $\sim 10^{4}-10^{5}$ depending on which characteristic length is used to define $\tau_{R}$ and $\tau_{A}$.

Figure 3 shows typical experimental results to date; these are photographs taken by a gated, intensified chargecoupled-device camera (Xybion model ISG-250) with $80 \mathrm{~ns}$ shutter speed. The time evolution is obtained by photographing a sequence of identical discharges with the camera trigger successively delayed by $\approx 200-600$ ns increments so as to make a pseudo-movie. This method is possible because good shot-to-shot reproducibility is obtained over a large range of operating conditions. The camera had a very limited gray scale (10 levels) and the exposure was adjusted for each shot to provide maximum information; however, the adjustment of camera exposure was not perfect which is why some shots appear overexposed (e.g., the $-0.1 \mu$ s photo). The horseshoe magnet is mounted on a $25.4 \mathrm{~cm}$ Conflat flange which in turn is mounted on a $20 \mathrm{~cm}$ diameter tubulation extending about $10 \mathrm{~cm}$ outward from the vacuum chamber wall. This configuration results in the prominence simulator being recessed about $10 \mathrm{~cm}$ in a $20 \mathrm{~cm}$ diameter depression relative to the vacuum chamber wall surface (this is not an optimum configuration and it is intended to eliminate the recessed geometry in future experiments).

The current and voltage have the following time behavior. After the ignitron switch connects the capacitor bank across the horseshoe magnet poles, the applied voltage remains at $6 \mathrm{kV}$ for $2 \mu \mathrm{s}$ and then abruptly drops to approximately $1 \mathrm{kV}$ in less than $0.1 \mu \mathrm{s}$. The applied voltage appears balanced across the electrodes, i.e., one electrode has $V / 2$ and the other has $-V / 2$ relative to the vacuum chamber wall. The time of abrupt voltage drop (collapse) is defined as $t$ $=0$ and presumably is when the plasma conductivity becomes sufficiently high for the plasma to act as a low impedance load (i.e., approximate short circuit) for the capacitor bank. Thus for $t<0$, the capacitor bank functions as a voltage source while for $t>0$ it functions as a current source. After $t=0$ the current increases sinusoidally to a peak value of $18 \mathrm{kA}$ at time $t=5.5 \mu \mathrm{s}$, then rings through zero at $t$ $=13 \mu \mathrm{s}$, and attains a peak reversed value of $-5 \mathrm{kA}$ at $t$ $=18 \mu \mathrm{s}$. It rings through zero once again at $t=25 \mu \mathrm{s}$, has a small positive peak of $2 \mathrm{kA}$ at $t=31 \mu \mathrm{s}$, and then dies without further ringing.

The first frame in Fig. 3 shows that an arch-shaped flux tube is initially formed between the magnet poles at times before the applied voltage collapses; the regular oval is the image of the $20 \mathrm{~cm}$ diameter depression. The flux tube becomes slightly twisted as the current ramps up. However, at $t \approx 1 \mu$ s (i.e., before the initial flux tube has a chance to become strongly twisted), secondary arcs develop between the magnet poles and the wall; these secondary arcs presumably follow the fringing fields. In hindsight, this turns out not to be surprising because there are voltage drops $V / 2$ between the magnet poles and the wall as well as the voltage drop $V$ between the magnet poles so that there is more than one possible circuit. The secondary arcs are quite interesting; they become twisted and have complicated, yet reproducible dynamics. After formation they become contorted, extend outwards, appear to merge near the axis, and then explode outwards. The $6.5 \mu$ s photo suggests braiding of the secondary arcs. The photos in Fig. 3 cover the time from just before voltage collapse to the first time the current rings through zero, and in particular, the photos for $t=4.8 \mu \mathrm{s}, 6.5 \mu \mathrm{s}$, and $7.0 \mu \mathrm{s}$ correspond to times when the current is within $5 \%$ of its maximum value of $18 \mathrm{kA}$. Finally, we note that when viewed from end-on, the simulated prominences are seen to have either an S shaped pattern or a comma pattern. 


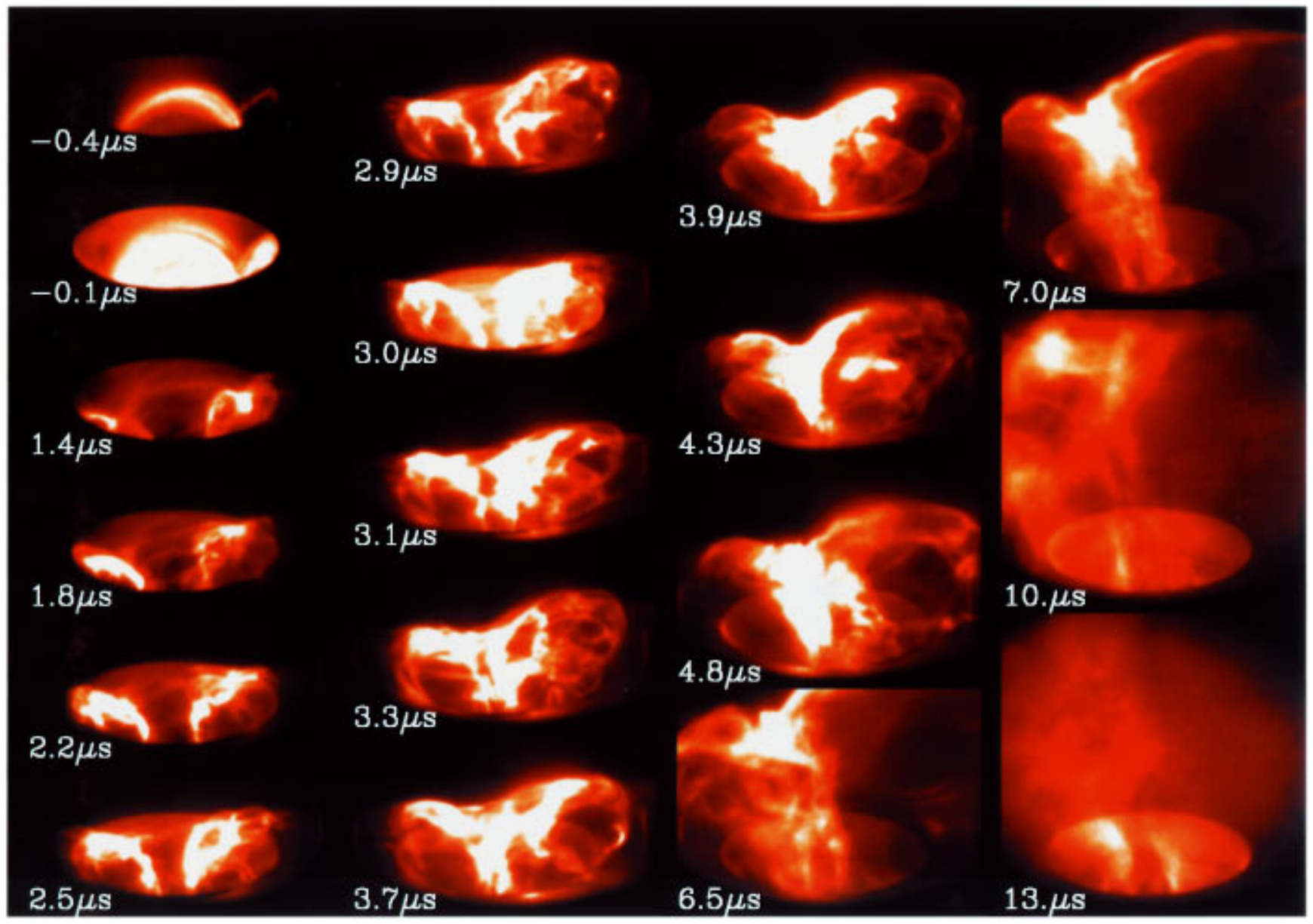

FIG. 3. Photographs showing typical evolution of the experimental configuration. The time $t=0$ denotes when the applied voltage suddenly drops towards zero, i.e., when the plasma conductivity becomes sufficiently high for the plasma to act like a nearly short-circuited load.

\section{INTERPRETATION OF THE EXPERIMENTAL RESULTS}

\section{A. Identification of the helicity sense}

The photographs show the projection onto a plane of three-dimensional twisted structures and at first sight, the handedness of these twisted structures is not obvious. In order to determine handedness we consider the most elementary property distinguishing the projection onto a plane of right- and left-handed twists. The two left curves in Fig. 4 show the projections onto a plane of left- and right-handed helices such as may be formed by spiralling ribbons about a center rod. The gross morphology of the projection of rightand left-handed helices is identical, but there are differences in detail. Both right- and left-handed helices can produce both forward and reversed $\mathrm{S}$ shapes depending on which helix portion is projected; for example, the bottom three segments of both right- and left-handed helices in Fig. 4 have a forward S projection, while the top three segments have a reverse $\mathrm{S}$ projection.

Figure 4 shows that the projection of a helix onto a plane consists of a sequence of up-sloping (//) and down-sloping (II) segments. The essential difference between the projection of right- and left-handed helices is manifested by how the up- and down-sloping segments overlap. For left-handed twist, the up-sloping segments lie below the down-sloping segments (mnemonic: up-beLow) while for right-handed twist, the up-sloping segments lie above the down-sloping segments (mnemonic: up-oveR). The two curves on the right of Fig. 4 thus constitute the fundamental elements of twist. To identify handedness, a photo should be rotated so that a segment of magnetic field has an up-slope and if this up-

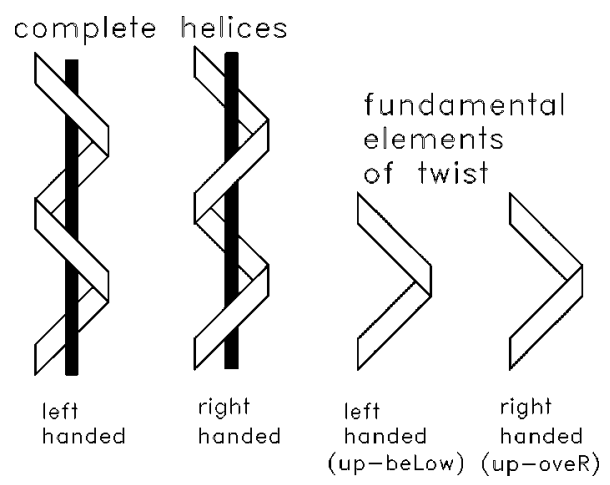

FIG. 4. Left: A pair of right- and left-handed helices show that the projection onto a plane is the same for both right- and left-handed helices. Right: Demonstration that the fundamental quantity determining twist is the order of overlap of up- and down-sloping ribbons. 


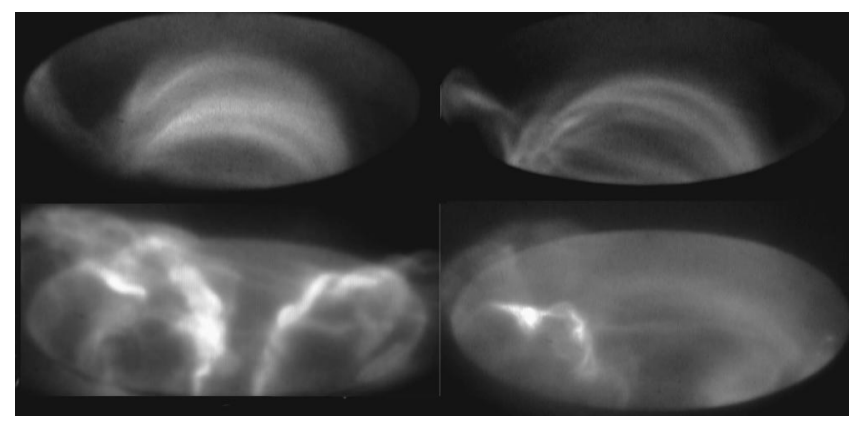

FIG. 5. Demonstration of the reversal of twist when the electromagnet polarity is reversed. The two photos on the left had the same electromagnet polarity as in Fig. 3. The two photos on the right were made at similar times, but had the electromagnet polarity reversed. Analysis of handedness using the identification method presented in Fig. 4 shows that the twists in the left-hand photos are left handed and twists in the right-hand photos are right handed.

sloped segment lies above its adjacent down-sloped segments at the point of overlap, the twist is right handed. If the up-sloped segment lies below its adjacent down-sloped segments at the point of overlap, the twist is left handed.

Figure 5 demonstrates that the experimentally observed handedness of twist does indeed reverse when the polarity of the horseshoe magnet is reversed (handedness is determined using the method described in the previous paragraph). The two photos on the left (top is early time, bottom is somewhat later) have left-handed twists and were obtained with the horseshoe magnet polarity the same as in Fig. 3. The two curves on the right were obtained with horseshoe magnet polarity reversed (again top is early time, bottom is somewhat later) and have right-handed twists.

\section{B. General force-free solution in infinite half-space}

To model the experiment we seek solutions to Eq. (1) in infinite half-space with uniform $\lambda$ and a prescribed $B_{z}$ on the ground plane $\left(z=0\right.$ surface). Nakagawa ${ }^{4}$ and Finn, Manheimer, and $\mathrm{Ott}^{17}$ found that a general solution to Eq. (1) could be expressed in terms of a single scalar function $\psi(\mathbf{r})$ which is neither a flux function nor a potential function, but has properties related to both. We find it convenient to write such a solution to Eq. (1) as

$$
\mathbf{B}=-\lambda \hat{z} \times \nabla \psi-\nabla \times(\hat{z} \times \nabla \psi),
$$

a form slightly different from Refs. 4 and 17 . Direct substitution of Eq. (3) into Eq. (1) shows that $\psi$ must satisfy the Helmholtz equation

$$
\nabla^{2} \psi+\lambda^{2} \psi=0,
$$

which in cylindrical geometry is

$$
\frac{1}{r} \frac{\partial}{r}\left(r \frac{\partial \psi}{\partial r}\right)+\frac{1}{r^{2}} \frac{\partial^{2} \psi}{\partial \theta^{2}}+\frac{\partial^{2} \psi}{\partial z^{2}}+\lambda^{2} \psi=0 .
$$

At this point we part company with traditional spheromak solutions which assume periodic behavior in the $z$ direction in order to satisfy wall boundary conditions at $z=0$ and $z$ $=h$. We instead specify boundary conditions on the ground plane $z=0$ and require $\psi$ to vanish as $z \rightarrow \infty$. To achieve this, an $\exp (-k z)$ dependence is assumed ${ }^{4,7}$ where $z$ is the elevation above the ground plane. The solution to Eq. (5) will therefore be composed of a linear superposition of modes of the form

$$
\psi_{m}(k, r, \phi, z)=\bar{\psi}_{m}(k) J_{m}(\kappa r) e^{-i m \phi-k z},
$$

where $\kappa=\sqrt{k^{2}+\lambda^{2}}$. For simplicity we consider the behavior of a single mode; this turns out not to be a serious restriction.

\section{Three-dimensional topology of field lines}

It is useful to express Eq. (3) as

$$
\mathbf{B}=-\lambda \hat{z} \times \nabla \psi+\nabla \frac{\partial \psi}{\partial z}+\hat{z} \lambda^{2} \psi
$$

using Eq. (4). In the limit $\lambda \rightarrow 0$, Eq. (7) gives $\mathbf{B}$ $=\nabla(\partial \psi / \partial z)$; i.e., the magnetic field becomes the gradient of a scalar function and so is a vacuum field consistent with the requirements of Eq. (1). The effect of finite $\lambda$ is twofold: first, finite $\lambda$ adds a correction to the $z$ component of the vacuum field; second, finite $\lambda$ adds the term $-\lambda \hat{z} \times \nabla \psi$.

Consider a contour map of $\psi$ in the $z=0$ plane. The contours show the topography of the hills/valleys of $\psi$ and also the neutral lines ( $\psi=0$ lines) between hills and valleys. For Eq. (6) the contour maps at different $z$ elevations will be isomorphic to the ground plane contour map, because $\psi$ is just attenuated by $\exp (-k z)$ on moving to higher elevations. The magnetic field for Eq. (6) will be

$$
\mathbf{B}=-\lambda \hat{z} \times \nabla \psi-k \nabla \psi+\hat{z} \lambda^{2} \psi
$$

so that the vacuum component (middle term of the righthand side) consists of potential field lines going from hills to valleys of $\psi$. If one considers a $\psi$ hill in the ground plane, then $-\lambda \hat{z} \times \nabla \psi$ points along a level contour of the hill. This $-\lambda \hat{z} \times \nabla \psi$ term is azimuthal with respect to the $z$ axis through the $r, \phi$ location of the hill summit, is proportional to $\lambda$, and injects a twist into the field lines as they go from hill to valley. The specific components of $\mathbf{B}$ are

$B_{r}=-\bar{\psi}\left[\frac{\lambda m}{r} J_{m}(\kappa r) \sin m \phi+k \kappa J_{m}^{\prime}(\kappa r) \cos m \phi\right] e^{-k z}$,

$B_{\phi}=-\bar{\psi}\left[\lambda \kappa J_{m}^{\prime}(\kappa r) \cos m \phi-\frac{m k}{r} J_{m}(\kappa r) \sin m \phi\right] e^{-k z}$,

$B_{z}=\kappa^{2} \bar{\psi} J_{m}(\kappa r) e^{-k z} \cos m \phi$.

The $z$ trajectory of a field line starting on the slope of a hill in the ground plane can be visualized as analogous to the trajectory of a particle that has an initial upwards $z$ velocity as it leaves the ground plane. Equations (9) show that the $B_{z}$ contours are identical in form to the $\psi$ contours; thus the $B_{z}$ contours at all elevations are isomorphic. Therefore $B_{z}$ changes sign when either $J_{m}(\kappa r)$ or $\cos m \phi$ changes sign; the zeros of $J_{m}$ and of $\cos m \phi$ are therefore the separatrices of the contours and also the neutral lines of $\psi, B_{z}$, and $J_{z}$ (i.e., where all these quantities change sign).

Because the contours are isomorphic at all elevations, one is tempted to think that a field line starting upwards from 
a specified $r, \phi$ in the ground plane where $B_{z}$ is positive would continue upwards indefinitely. This does not happen in general because the $B_{r}$ and $B_{\phi}$ components deflect all field line trajectories radially and azimuthally from their initial $r, \phi$ coordinates (only the unique field line lifting off from a hill summit would have no horizontal deflection and go up forever). The horizontal deflection eventually causes a typical upbound field line to cross a neutral line and pass into a region where $B_{z}$ is negative. Since the vertical trajectory of the field line is proportional to $B_{z}$, the field line now heads down back towards the ground plane, again being deflected by $B_{r}$ and $B_{\phi}$ components. Thus arch-shaped, twisted, field lines are intrinsic to the solution given in Eq. (6).

An important topological consequence of Eqs. (9) is that the field lines associated with Eqs. (9) cannot crossover each other; i.e., the projection of field lines on the ground plane will never have an $\mathrm{x}$ shape from a crossover (as in a knot or as in a flux tube at high altitude crossing over another flux tube at low altitude). This restriction can be understood by considering what would happen if a field line at elevation $z_{2}$ did cross over a field line at elevation $z_{1}$. Such a cross-over would require the perpendicular components $\mathbf{B}_{\perp}=B_{r} \hat{r}$ $+B_{\phi} \hat{\phi}$ of two field lines at the same $r, \phi$ but different $z$ to be nonparallel; but this is not allowed since Eqs. (9) constrains the ratio $B_{r} / B_{\phi}$ to be independent of $z$ because for the single $k$ assumed here, the $\exp (-k z)$ dependence cancels from the ratio $B_{r} / B_{\phi}$.

This noncrossing property is a consequence of restricting the solution to a single $k$ mode. Crossing would be allowed if there were a superposition of modes with different $k$ because then the $\exp (-k z)$ factors would not cancel from $B_{r} / B_{\phi}$ making this ratio $z$ dependent. The $\mathrm{S}$ shapes routinely observed on the sun thus presumably involve just a single $\exp (-k z)$ mode, because an $\mathrm{S}$ shaped projection is consistent with noncrossing. We note that solutions of Eq. (1) with dependence other than a single $\exp (-k z)$ mode have been shown to exhibit crossings. For example, solutions subject to three-dimensional box boundary conditions have been examined by Finn et al. ${ }^{18}$ and have been shown to allow crossings (e.g., knots) while solutions expressed in terms of spherical coordinates have been shown by Lites et al. ${ }^{19}$ to involve crossings and knots also. The crossings in these situations occur because both box boundary conditions and solutions in spherical coordinates should be equivalent to superimposing a $k$ spectrum of the modes given in Eq. (9).

\section{Approximate solutions relevant to the experiments}

The horseshoe plasma gun used in the experiments may be considered as a source which prescribes an $m=1$ ground plane boundary condition for $B_{z}$; i.e., the experimental configuration is approximated by

$$
\psi=\bar{\psi} J_{1}(\kappa r) \cos \phi \exp \left(-z \sqrt{\kappa^{2}-\lambda^{2}}\right)
$$

providing $|\lambda|<\kappa$. Because the amplitude of the Bessel function decreases for large $r$, we focus attention on the first complete oscillation of $J_{1}$, namely the region from $r=0$ to the second root of the Bessel function. The magnet poles provide local maxima of oppositely directed $B_{z}$ field in the ground plane and can be considered as the innermost hillvalley pair of $B_{z}$ maxima and minima and are surrounded by a circular neutral line corresponding to the first root of $J_{1}$. Outside this neutral line there is a second, oppositely directed, geometrically larger but weaker amplitude hill-valley pair corresponding to the fringing fields going from one magnet pole to the other the long way around the horseshoe. As a simplifying approximation, we ignore Bessel oscillations beyond the first full period of $J_{1}(\kappa r)$; this corresponds to a modest error in the large $r$ boundary conditions.

Since $J_{1}$ has its first maximum when $\kappa r \approx 1.8$, if $L$ is the separation between magnet poles, then $\kappa L / 2 \simeq 1.8$ or $\kappa$ $\simeq 3.6 / L$. The solution given by Eq. (10) fails when $|\lambda|>\kappa$ suggesting that stable, attached, force free-states cannot exist when $|\lambda|>\kappa$. Integrating Eq. (1) over the $z=0$ surface encompassing the hill corresponding to the $+B_{z}$ magnet pole gives

$$
\int d s \hat{z} \cdot \nabla \times \mathbf{B}=\lambda \int d s \hat{z} \cdot \mathbf{B}
$$

so that $\lambda=\mu_{0} I / \Phi$ where $I$ is the total current flowing from the magnet pole up to the neutral line and $\Phi$ is the total flux up to the neutral line. The condition for stable, attached force-free states will be $|\lambda|<3.6 / L$. Instability should occur when

$$
\left|\frac{\mu_{0} I}{\Phi}\right|>\frac{3.6}{L}
$$

a condition analogous to the spheromak formation condition. $^{20,21}$ For the experiments here, $L \simeq 0.12 \mathrm{~m}, \Phi$ $\simeq 0.3 \mathrm{mWb}$ so this model predicts instability when $I>7 \mathrm{kA}$. The experimental current was increasing sinusoidally from near zero at $t=0$ to a maximum of $18 \mathrm{kA}$ at $t=6.5 \mu \mathrm{s}$. Thus the magnitude of the experimental current is in reasonable agreement with the model.

\section{E. Bifurcation and destruction of flux tubes when $\lambda$ increases}

We have investigated the magnetic topology associated with Eq. (10) using direct numerical calculation of the field line trajectories

$$
\frac{d \mathbf{r}}{d s}=\frac{\mathbf{B}}{B},
$$

where $\mathbf{B}$ is prescribed by Eqs. (9) and $s$ is the path length along the field line. Contrary to conventional wisdom, we find that flux tubes are a somewhat artificial concept compared to field lines because given a starting point $r, \phi$ in the ground plane, a unique field line may always be calculated by numerical integration of Eq. (13), whereas given a starting surface of intersection in the ground plane, a unique well-defined flux tube may not always exist.

The attempt to construct a flux tube proceeds as follows: An $n$-sided polygon is prescribed in the ground plane; this polygon defines the intersection of the proposed flux tube with the ground plane. From a more detailed point of view, the polygon vertices $\left(r_{i}, \phi_{i}\right)$ define the intersection with the 
ground plane of selected field lines on the flux tube surface and, in particular, pairs of adjacent vertices $\left(r_{i}, \phi_{i ;} r_{i+1}, \phi_{i+1}\right)$ define the intersection with the ground plane of the ribbon surface between two adjacent field lines. A typical ribbon rises from the ground plane, reaches its maximum $z$, then turns back and heads downward to intercept the ground plane at some other location $\left(r_{i}^{\prime}, \phi_{i ;}^{\prime} r_{i+1}^{\prime}, \phi_{i+1}^{\prime}\right)$. If $\lambda$ is finite, the ribbon will twist. If the flux tube exists, its surface will be the sum of the surfaces of these ribbons. The field line starting from the center of the polygon defines the magnetic axis of the polygonal flux tube. For $\lambda=0$, the set of ribbons form an untwisted flux tube going from a hill to a valley. However, as $\lambda$ is increased the flux tube twists up and its magnetic axis also rotates, deviating from its initial trajectory.

The flux tube concept gives difficulties because as $\lambda$ is increased and the magnetic axis swerves from its initial trajectory, a particular field line starting from point $\left(r_{i}, \phi_{i}\right)$ might be sufficiently deflected so as to cross a different $\psi$ neutral line from the other field lines. In this case, the ribbon for which this field line acted as a perimeter will cease to exist, because the field line starting from $\left(r_{i}, \phi_{i}\right)$ will have a completely different trajectory from the assumed nearly parallel field line starting from $\left(r_{i+1}, \phi_{i+1}\right)$; from a mathematical point of view, the field lines are no longer analytic. As $\lambda$ is increased further, two adjacent field lines will cross a different separatrix (neutral line), resulting in a complete ribbon following a quite different trajectory from all the other ribbons. Thus the various ribbons comprising the intended flux tube do not all cross the same separatrix (neutral line) and what was intended to be a single flux tube actually bifurcates into smaller flux tubes. The downward parts of the bifurcated flux tubes intersect the ground plane at distinct, highly separated locations. (We find that this bifurcation does not occur if $m=0$ symmetry is used because $m=0$ symmetry has only one kind of separatrix, the zeroes of the Bessel function.)

Bifurcation suggests serious problems with the line-tying concept; i.e., the concept that field lines intercepting a fluxconserving conducting surface are permanently anchored to the point of interception. Contrary to the line-tying concept, we find from direct numerical integration of field lines that the ends of individual field lines are not tied to fixed points in the flux-conserving conducting surface. We impose the same $B_{z}$ field in the ground plane for different values of $\lambda$; this corresponds to having a fixed flux intersecting the ground plane, but varying the current flowing along the field lines. From Eqs. (9) and (10) it is seen that $\lambda$ may be varied without changing $B_{z}$ in the ground plane. Our numerical integration starts at a prescribed initial point $r_{i}, \phi_{i}$ in the ground plane, and then integrates Eq. (13) with fields given by Eqs. (9) to follow the field line as it rises to its maximum $z$ and then falls back to the ground plane. We find that the landing point $\left(r_{i}^{\prime}, \phi_{i}^{\prime}\right)$ where the falling field line intersects the ground plane is not fixed, but depends on $\lambda$. Bifurcation of a flux tube leads to a discontinuous jump in the landing point. Thus while flux remains conserved in the ground plane when $\lambda$ is varied, the two ends of a field line are not tied to fixed points.

Figure 6 shows two numerically calculated sets of rib- bons between field lines for fixed $\kappa$ with $\lambda / \kappa$ varying from 0 to -0.97 . It is seen that the ribbon surfaces twist, rotate, and sometimes fail to exist as $\lambda$ increases. Also, the ribbons extend to large $z$ as $|\lambda|$ increases, consistent with $k$ $=\sqrt{\kappa^{2}-\lambda^{2}} \rightarrow 0$ as $|\lambda| \rightarrow \kappa$. The ribbons were chosen so as to simulate the experiment: one proposed flux tube (set of red/ blue ribbons) is calculated using forward integration of Eq. (13) with the starting point being a small polygon on a hill (positive magnet pole), while the other proposed flux tube (green/purple) is calculated using backwards integration with the starting point being a similar small polygon on a valley (negative magnet pole). The projection of the ribbons on the ground plane is also shown (red for red/blue set, green for green/purple set); for clarity, this projection on the ground plane is repeated in planar view in Fig. 7. At small $\lambda$ both sets of ribbons behave according to conventional wisdom; i.e., the red/blue ribbons form a flux tube which extends from the primary hill to the primary valley while the green/purple ribbons form a flux tube which goes from primary valley to primary hill. When $|\lambda / \kappa|$ increases to 0.60 , the ribbons start to swerve so that instead of crossing the separatrix where $\cos \phi$ vanishes (i.e., the line $\phi= \pm \pi / 2$ ) the ribbons successively cross the circular separatrix $\kappa r=3.8$ (i.e., where $J_{1}$ vanishes). As $|\lambda / \kappa|$ increases past 0.91 the ribbons cross the $\phi= \pm \pi / 2$ separatrix again. The forward and backward integrated field lines become interlaced after swerving $360^{\circ}$. Even though the field lines are interlaced they do not have crossovers as can be seen from their projections on the ground plane. Also after swerving $360^{\circ}$, the ribbons cross through (penetrate) each other, showing that the ribbon surfaces can no longer be the surfaces of flux tubes. Thus flux tubes and flux surfaces cease to exist at large $\lambda$. Nevertheless, the system has the interesting topological property that when viewed from above (i.e., viewing the projection onto the ground plane), the field lines do not overlap, but when viewed from the side the field lines appear twisted.

We believe that the formation of the secondary arcs in our experiment is a manifestation of this topological evolution. The ground plane $B_{z}$ contours for the horseshoe magnet consist of a primary hill/valley pair (corresponding to the two magnet poles) and a secondary hill/valley pair (corresponding to the fringing fields going the long way around the horseshoe). When $\lambda$ is small, a flux tube starting on the small $r$ side of the primary hill arches straight over to the primary valley, i.e., starts on one magnet pole and has a landing point on the other. However, as $|\lambda|$ is increased, the flux tube swerves and the projection of its magnetic axis on the ground plane rotates. At a critical $|\lambda|$ the rotation is such that the outermost portion of the original flux tube will have a landing point on the secondary valley (i.e., where the fringing field intersects the ground plane), and for sufficiently large $|\lambda|$, the entire flux tube goes to the secondary valley. This behavior is quite evident in the numerical calculations and appears topologically consistent with the development and evolution of the secondary arcs shown in Fig. 3. In particular, the $6.5 \mu$ s data suggests braiding and interleaving of ribbons in a manner qualitatively similar to the numerical calculations. 


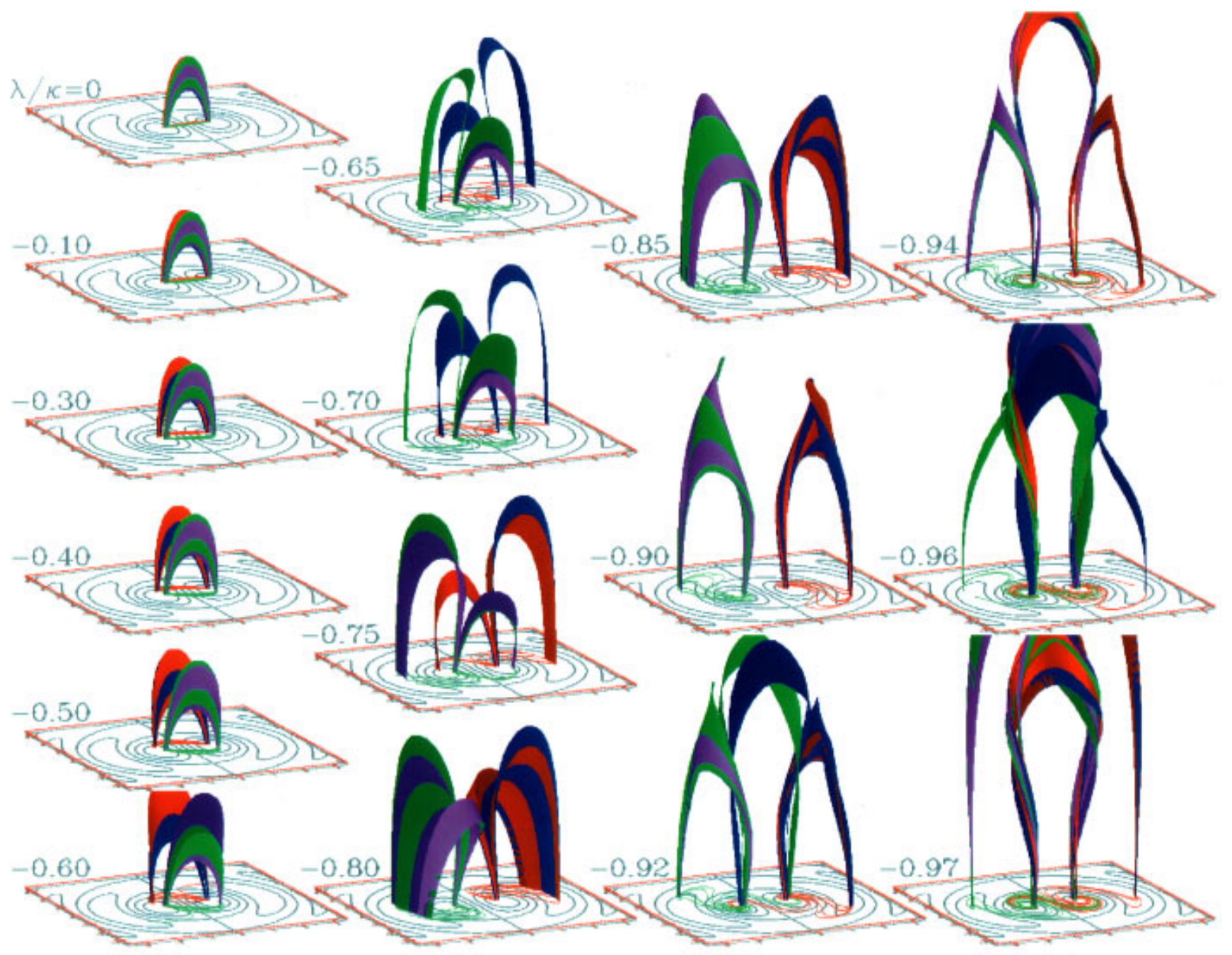

FIG. 6. Field lines, ribbons (when existing), and flux tubes (when existing) obtained from direct numerical integration of Eq. (13) using Eqs. (9). Contours of constant $\psi$ (also constant $B_{z}, J_{z}$ ) in ground plane are shown as well as projection of field lines onto ground plane.

\section{F. Explanation for the $S$ shapes}

The projection of these twisted flux tubes onto the ground plane has the forward or backward S shape characteristic of photographs of solar prominences projected onto the solar surface (backward S for Figs. 6 and 7, since $\lambda$ was chosen negative). Rust and Kumar ${ }^{13}$ proposed that the $S$ shape could be explained in terms of a kink instability acting on a straight flux tube. Although their explanation does give an $\mathrm{S}$ shape, it does not explain why the observations always show only one full $\mathrm{S}$ and not a nonintegral number of S's such as would occur by choosing an arbitrary length for the assumed kinked flux tube. Furthermore, as seen in Fig. 4, the projections of both left- and right-handed helices can be forward or reversed $S$ shapes depending on which segments of the helix are selected. We propose here a somewhat different explanation from that in Ref. 13 for the cause of the $\mathrm{S}$ shape; our explanation shows that there should be exactly one S, just as is observed.

The essence of our explanation lies in Eq. (1). If $\lambda$ is positive then $\mathbf{J}$ and $\mathbf{B}$ are parallel whereas if $\lambda$ is negative, then $\mathbf{J}$ and $\mathbf{B}$ are antiparallel. Consider an equilibrium with positive $\lambda$ and follow a field line rising from a $B_{z}$ hill in the ground plane. By assumption $B_{z}$ is positive and since $\lambda$ is positive, $J_{z}$ is also positive. Since $\mu_{0} J_{z}=\hat{z} \cdot \nabla \times \mathbf{B}$ the projection of $\mathbf{B}$ onto any plane parallel to the ground plane must have a positive curl (counterclockwise motion of projection of field line onto $z$ plane). After the magnetic field line reaches its maximum $z$ elevation, it heads downwards so that both $B_{z}$ and $J_{z}$ will be negative. Thus $\hat{z} \cdot \nabla \times \mathbf{B}$ is a negative curl (clockwise motion). The sum of the projections of the upward and downward trajectories gives a forward $\mathrm{S}$ shape. If $\lambda$ is negative, then the upward trajectory has a negative curl projection, while the downward projection has a positive curl projection, leading to a reverse $\mathrm{S}$ shape as is evident in Figs. 6 and 7. This also shows that the point of inflection (where the curl vanishes) of the $\mathrm{S}$ shape is the turning point (maximum altitude, $B_{z}=0, J_{z}=0$ ) of the field line and also defines the separatrix (neutral line) between positive and negative magnetic polarity. 

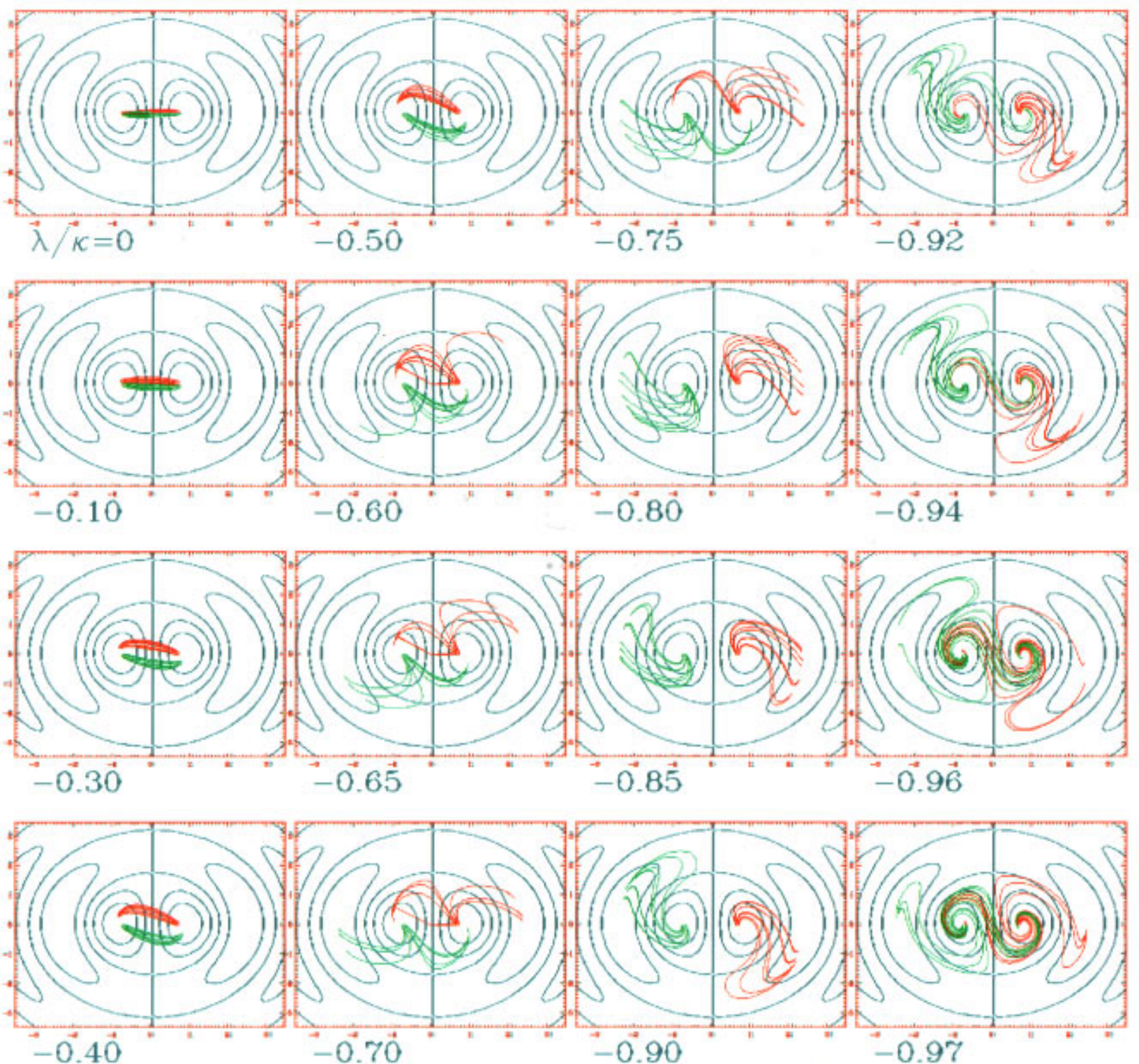

FIG. 7. Projection onto the ground plane (planar view) of the field lines in Fig. 6. Contours of $\psi$ are also shown. Note the backwards S shape of the projected field lines at large $|\lambda|$; the backwards direction is consistent with the negative polarity of the $\lambda$ 's used for these plots (forward S shapes are obtained if the $\lambda$ 's have positive polarity).

\section{SUMMARY AND CONCLUSIONS}

The physics of prominence eruption and spheromak formation have been shown to be quite similar so that spheromak technology can be exploited to create reasonable simulations of solar prominences providing solar boundary conditions and geometry are approximated. Photos of simulated prominences show morphology similar to actual solar prominences, especially twist, instability, and bifurcation. A theoretical model predicts instability thresholds and topological behavior in qualitative agreement with the observations. This model also provides insights regarding the threedimensional topology of magnetic fields above the solar surface, suggests there are difficulties with both the flux tube concept and the line-tying concept, and provides an explanation for the characteristic $S$ shape of prominences. Future experiments will address behavior when $\lambda$ exceeds $\kappa$ (pre- sumably eruption and reconnection) and also what happens when the fringing fields are insulated so that the current is forced to flow between the primary hills and valleys (magnet poles) only.

\section{ACKNOWLEDGMENTS}

We wish to thank F. C. Cosso for assistance with vacuum and electronics and J. Yee for assistance with operation of the high-speed camera.

This work was supported by the National Science Foundation and the United States Department of Energy.

${ }^{1}$ E. Tandberg-Hanssen, The Nature of Solar Prominences (Kluwer Academic, Dordrecht, 1995).

${ }^{2}$ D. M. Rust and A. Kumar, Astrophys. J. 464, L199 (1996).

${ }^{3}$ B. Vrsnak, V. Ruzdak, and B. Rompolt, Sol. Phys. 136, 151 (1991). 
${ }^{4}$ Y. Nakagawa, M. A. Raadu, D. E. Billings, and D. McNamara, Sol. Phys. 19, 72 (1971).

${ }^{5}$ J. B. Taylor, Phys. Rev. Lett. 33, 1139 (1974).

${ }^{6}$ J. B. Taylor, Rev. Mod. Phys. 58, 741 (1986).

${ }^{7}$ J. Heyvaerts and E. R. Priest, Astron. Astrophys. 137, 63 (1984).

${ }^{8}$ P. K. Browning, J. Plasma Phys. 40, 263 (1988).

${ }^{9}$ D. M. Rust and A. Kumar, Sol. Phys. 155, 69 (1994).

${ }^{10}$ L. F. Burlaga, J. Geophys. Res. 93, 7217 (1988).

${ }^{11}$ D. M. Rust, Geophys. Res. Lett. 21, 241 (1994).

${ }^{12}$ M. Gigolashvili, Sol. Phys. 60, 293 (1978); S. F. Martin, W. H. Marquette, and R. Bilimoria, Astron. Soc. Pac. Conf. Ser. 27, 53 (1992); A. A. Pevtsov, R. C. Canfield, and T. R. Metcalf, Astrophys. J. 440, 109 (1994).

${ }^{13}$ D. M. Rust and A. Kumar, Astrophys. J. 464, L199 (1996).

${ }^{14}$ L. Woltjer, Proc. Natl. Acad. Sci. USA 44, 489 (1958).

${ }^{15}$ T. R. Jarboe, I. Henins, H. W. Hoida, R. K. Linford, J. Marshall, D. A. Platts, and A. R. Sherwood, Phys. Rev. Lett. 45, 1264 (1980); W. C.
Turner, G. C. Goldenbaum, E. H. A. Granneman, J. H. Hammer, C. W. Hartman, D. S. Prono, and J. Taska, Phys. Fluids 26, 1965 (1983); G. C. Goldenbaum, J. H. Irby, Y. P. Chong, and G. W. Hart, Phys. Rev. Lett. 44, 393 (1980); M. R. Brown and P. M. Bellan, ibid. 64, 2144 (1990); Y. Ono, A. Morita, M. Katsuari, and M. Yamada, Phys. Fluids B 5, 3691 (1993); A. al-Karkhy, P. K. Browning, G. Cunningham, S. J. Gee, and M. G. Rusbridge, Phys. Rev. Lett. 70, 1814 (1993).

${ }^{16}$ P. K. Loewenhartdt, M. R. Brown, J. Yee, and P. M. Bellan, Rev. Sci. Instrum. 66, 1050 (1995).

${ }^{17}$ J. M. Finn, W. M. Manheimer, and E. Ott, Phys. Fluids 24, 1336 (1981). ${ }^{18}$ J.M. Finn, P. N. Guzdar, and D. Usikov, Astrophys. J. 427, 475 (1994).

${ }^{19}$ B. W. Lites and B. C. Low, Sol. Phys. 174, 91 (1997).

${ }^{20}$ M. J. Schaffer, Phys. Fluids 30, 160 (1987).

${ }^{21}$ C. W. Barnes, T. R. Jarboe, G. J. Marklin, S. O. Knox, and I. Henins, Phys. Fluids B 2, 1871 (1990). 